\title{
Etiology of Dementia in Thai Patients
}

\author{
Pornpatr A. Dharmasaroja ${ }^{a}$ b Jintana Assanasen ${ }^{c}$ Sunsanee Pongpakdee ${ }^{d}$

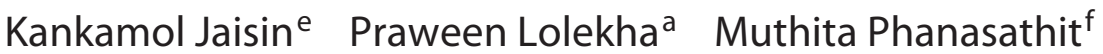

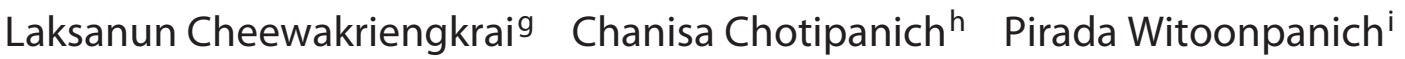 \\ Sutisa Pitiyarn ${ }^{j}$ Pongtawat Lertwilaiwittaya ${ }^{k}$ Charungthai Dejthevaporn ${ }^{i}$ \\ Chanin Limwongse $^{k}$ Kammant Phanthumchindal The Collaborative \\ Aging and Dementia Research Society Thailand (CART)
}

\begin{abstract}
a Department of Medicine, Faculty of Medicine, Thammasat University, Pathumthani, Thailand; ${ }^{b}$ Faculty of Medicine, Chulabhorn International College of Medicine, Thammasat University, Pathumthani, Thailand; ' ${ }^{\circ}$ Division of Geriatric Medicine, Department of Medicine, Faculty of Medicine, Siriraj Hospital, Mahidol University, Bangkok, Thailand; ${ }^{\mathrm{d}}$ Department of Medicine, Bhumibol Adulyadej Hospital, Royal Thai Airforce, Bangkok, Thailand; ${ }^{\text {e Department of }}$ Psychiatry, Faculty of Medicine, Siriraj Hospital, Mahidol University, Bangkok, Thailand; ${ }^{f}$ Department of Psychiatry, Faculty of Medicine, Thammasat University, Pathumthani, Thailand; ${ }^{9}$ Department of Medicine, Phramongkutklao Hospital, Bangkok, Thailand; ' hational Cyclotron and PET Centre, Chulabhorn Hospital, Chulabhorn Royal Academy, Bangkok, Thailand; 'Division of Neurology, Department of Medicine, Faculty of Medicine, Ramathibodi Hospital, Mahidol University, Bangkok, Thailand; ' Medicine and Psychiatry Nursing Division, Department of Nursing, Siriraj Hospital, Mahidol University, Bangkok, Thailand; ${ }^{\mathrm{k}}$ Division of Medical Genetics, Department of Medicine, Faculty of Medicine, Siriraj Hospital, Mahidol University, Bangkok, Thailand; 'Department of Medicine, Chulalongkorn University, Bangkok, Thailand
\end{abstract}

\section{Keywords}

Etiology $\cdot$ Dementia $\cdot$ Thai $\cdot$ Asian

\begin{abstract}
Introduction: Molecular imaging has been developed and validated in Thai patients, comprising a portion of patients in the dementia registry. This should provide a more accurate diagnosis of the etiology of dementia, which was the focus of this study. Methods: This was a multicenter dementia study. The baseline characteristics, main presenting symptoms, and results of investigations and cognitive tests of the patients were electronically collected in the registry. Functional imaging and/or molecular imaging were performed in patients with an equivocal diagnosis of the causes of dementia, especially in atypical dementia or young onset
\end{abstract}

karger@karger.com www.karger.com/dee

Karger $\stackrel{\text { ' }}{=}$
(C) 2021 The Author(s)

Published by S. Karger AG, Basel

This is an Open Access article licensed under the Creative Common Attribution-NonCommercial-4.0 International License (CC BY-NC) (http://www.karger.com/Services/OpenAccessLicense), applicable to the online version of the article only. Usage and distribution for commercial purposes requires written permission. dementia (YOD). Results: There were 454 patients in the study. The mean age of the patients was 78 years, with $60 \%$ female. Functional imaging and/or molecular imaging were performed in 57 patients (57/454 patients, 13\%). The most common cause of dementia was Alzheimer's disease (AD; $50 \%)$, followed by vascular dementia (VAD; 24\%), dementia with Lewy bodies (6\%), Parkinson's disease dementia (6\%), frontotemporal dementia (FTD; $2.6 \%$ ), progressive supranuclear palsy (2\%), multiple system atrophy $(0.8 \%)$, and corticobasal syndrome (0.4\%). YOD accounted for 17\% (77/454 patients), with a mean age of 58 years. The causes of YOD were early onset amnestic AD (44\%), VAD (16\%), behavioral variant FTD (8\%), posterior cortical atrophy (6.5\%), and logopenic variant primary progressive aphasia (5.2\%). Conclusion: $A D$ was the most common cause of dementia in Thai patients and the distribution of other types of dementia and 
main presenting symptoms were similar to previous reports in Western patients; however, the proportion of YOD was higher.

(c) 2021 The Author(s)

Published by S. Karger AG, Basel

\section{Introduction}

The increased aging population is leading to a rising number of dementia patients. The Global Burden of Diseases, Injuries, and Risk Factors (GBD) Study 2016 assessed the global prevalence, mortality, and overall burden of dementia in 195 countries from 1990 to 2016, revealing an increase in individuals who lived with dementia from 20.2 million in 1990 to 43.8 million in 2016. Dementia is the fifth leading cause of death, and also negatively affects families and healthcare systems globally [1]. Thailand has been classed as an aging society since 2002, when the proportion of elderly reached more than $10 \%$ of the population [2]. The prevalence of dementia was $2.35-3.1 \%[2,3]$.

The most common cause of dementia is Alzheimer's disease (AD; 50-75\%), followed by vascular dementia (VAD; 20\%), dementia with Lewy bodies (DLB; 5\%), and frontotemporal lobar dementia (FTLD; 5\%); however, most data were from the Western countries [4, 5]. Following advances in biomarker and genetic marker development, diagnosis of the etiology of dementia can be made with more confidence. Moreover, in order to discover interventions that prevent, delay, or modify the course of the disease, a preclinical diagnosis is needed. Thus, biomarker criteria are included in the diagnosis criteria of dementia, especially in the research framework. Whether adding biomarker information would change the proportion of causes of dementia is still in doubt.

A study in Thailand reported the following causes of dementia in 207 patients: AD 55\%, VAD 20\%, mixed dementia 14\%, Parkinson's disease dementia (PDD) $10 \%$, and frontotemporal dementia (FTD) 1\% [6]. However, it was conducted in a single center without any neuroimaging biomarkers. The Collaborative Aging and Dementia Research Society Thailand (CART) is a dementia research working group comprised of experts in dementia from 4 university hospitals and 2 large tertiary care hospitals. Molecular imaging has been developed and validated in Thai patients and was performed in a portion of patients in the CART registry. This should provide a more accurate diagnosis of the etiology of dementia. The purpose of this study was to study the causes of dementia in Thai patients.

\section{Materials and Methods}

This was a cross-sectional, multicenter CART study carried out in 2018-2019. The baseline characteristics, main presenting symptoms, and results of investigations and cognitive function tests of the patients were electronically collected in the CART dementia registry using a standard consensus case report form.

Dementia was defined as progressive cognitive decline leading to interference with the activities of daily living. Young onset dementia (YOD) was classified if the onset of symptoms began at a younger than 65 years. The Thai Mental State Examination (TMSE) and Montreal Cognitive Assessment - Thai version (MOCA) were used to evaluate global cognitive function, with the cutoff point of 24/23 of TMSE and 25/24 of MOCA to define normal cognition/ cognitive impairment. Blood chemistry and at least 1 modality of neuroimaging (CT or MRI dementia protocol of the brain) were performed in all patients to exclude medical and surgical treatable causes. Whole brain ${ }^{18} \mathrm{~F}$-fluorodeoxyglucose positron emission tomography (FDG PET), ${ }^{11} \mathrm{C}$-Pittsburgh compound B positron emission (PiB PET), ${ }^{18} \mathrm{~F}-\mathrm{THK}-5351$ positron emission (Tau PET) and/or technetium-99m TRODAT-1 single-photon emission CT (TRODAT SPECT) were performed in patients with an equivocal diagnosis of the causes of dementia or to confirm the diagnosis, especially in atypical dementia or YOD. The causes of dementia were diagnosed by trained neurologists, psychiatrists, and geriatrists using standard criteria [7-15].

Descriptive analysis was conducted using the mean \pm SD for continuous variables and frequencies/percentages for categorical variables. Baseline characteristics, TMSE, MOCA scores, and the main presenting symptoms were compared between patients with amnestic AD dementia and VAD, DLB, PDD, or FTD (including the behavioral variant of FTD, nonfluent agrammatic variant primary progressive aphasia [PPA], and semantic variant PPA). Differences between groups were assessed by the $\chi^{2}$ test for categorical variables and the independent-samples $t$ test for continuous variables. The study was approved by the Human Research Ethics Committee of Thammasat University No. 1 (Faculty of Medicine), project No. MTU-EC-IM-0-213/60. Consent was informed and signed by the rightful relatives, and the Ethics Committee approved this consent procedure.

\section{Results}

The study comprised a total of 454 patients. The baseline characteristics of the patients are presented in Tables 1 and 2. The mean age of the patients was 78 years, and $60 \%$ were female. The mean duration of education was 8.5 years. The mean TMSE and MOCA scores were 17 and 14. Vascular risk factors were common in the patients. The most common cause of dementia was $\mathrm{AD}$ (50\%), followed by VAD (24\%), DLB (6\%), PDD (6\%), FTD (2.6\%), progressive supranuclear palsy (PSP; $2 \%$ ), multiple system atrophy $(0.8 \%)$, and corticobasal syndrome (CBS; 0.4\%). 
Table 1. Baseline characteristics of the study patients $(n=454)$

\begin{tabular}{lc}
\hline Age at diagnosis, years & $77.6 \pm 8.6$ \\
Sex & $181(40)$ \\
Male & $273(60)$ \\
Female & $8.5 \pm 5.4$ \\
Education, years & $17.4 \pm 6.2$ \\
TMSE score & $13.8 \pm 5.5$ \\
MOCA score & $326(72)$ \\
Hypertension & $131(29)$ \\
Diabetes mellitus & $372(82)$ \\
Hyperlipidemia & $87(19)$ \\
Old ischemic stroke & $27(6)$ \\
Coronary artery disease & $5(1)$ \\
Smoking & $17(4)$ \\
Atrial fibrillation & $140(31)$ \\
Family history of dementia & \\
\hline Diagnosis & \\
AD and variant & $176(39)$ \\
Late onset amnestic AD & $35(8)$ \\
Early onset amnestic AD & $9(2)$ \\
LV PPA & $5(1)$ \\
PCA & $111(24)$ \\
VAD & \\
Movement disorder-related dementia & $29(6)$ \\
DLB & $28(6)$ \\
PDD & $10(2)$ \\
PSP & $4(0.8)$ \\
MSA & $2(0.4)$ \\
CBS & $11(2)$ \\
Brontotemporal dementia and variant & $2(0.4)$ \\
Nonfluent agrammatic variant PPA & $1(0.2)$ \\
Semantic variant PPA & \\
Other causes of dementia & \\
Mixed dementia & \\
\hline
\end{tabular}

Data are presented as the mean $\pm \mathrm{SD}$ or $n(\%)$. AD, Alzheimer's disease; VAD, vascular dementia; LV, logopenic variant; PPA, primary progressive aphasia; PCA, posterior cortical atrophy; DLB, dementia with Lewy bodies; PDD, Parkinson's disease dementia; PSP, progressive supranuclear palsy; MSA, multiple system atrophy; CBS, corticobasal syndrome; FTD, frontotemporal dementia.

YOD accounted for $17 \%$ (77/454 patients), and the mean age of onset was 58 years. Forty-nine percent of patients were female. The mean MOCA score was 14.6. The main chief complaints were memory impairment (51\%), behavioral problems (15\%), motor problems (13\%), language problems (7\%), executive impairment (5\%), neuropsychiatric symptoms (4\%), and visuospatial impairment (4\%). The causes in this subgroup of patients were early onset amnestic AD (34/77 patients, 44\%), VAD (12 pa- tients, $16 \%$ ), behavioral variant FTD (6 patients, $8 \%$ ), posterior cortical atrophy (PCA; 5 patients, 6.5\%), logopenic variant (LV) PPA; 4 patients, 5.2\%), PSP (3 patients, $3.9 \%)$, multiple system atrophy (2 patients, $2.6 \%)$, PDD (2 patients, $2.6 \%)$, DLB (2 patients, $2.6 \%$ ), semantic variant PPA (1 patient, 1.3\%), familial prion disease (1 patient, $1.3 \%$ ), and unknown causes (5 patient, $6.5 \%$ ).

Functional imaging and/or molecular imaging were performed in 57 patients (57/454 patients, $13 \%$ ), which consisted of FDG PET in 52 patients, PiB PET in 46 patients, Tau PET in 31 patients, and TRODAT SPECT in 12 patients. The diagnoses of these patients were late onset $\mathrm{AD}$ (11 patients), early onset $\mathrm{AD}$ (9 patients), behavioral variant FTD (7 patients), PSP (6 patients), DLB (5 patients), PCA (5 patients), LV PPA (3 patients), VAD (3 patients), multiple system atrophy (2 patients), suspected non-AD pathology (2 patients), nonfluent agrammatic variant PPA (1 patient), PDD (1 patient), CBS (1 patient), and familial prion disease (1 patient).

Patients with VAD, as compared to those with $A D$, were older ( 75 vs. 72 years, $p=0.012$ ), a lower proportion were female ( 54 vs. $67 \%, p$ value $=0.023$ ), and a higher proportion had hyperlipidemia (96 vs. $77 \%, p<0.001$; Table 3). Patients with DLB had higher mean TMSE scores as compared to AD (21 vs. $17, p=0.009)$. Patients with PDD were older (76 vs. 72 years, $p=0.027$ ), and had higher TMSE scores (20 vs. $17, p=0.039$ ). Patients with FTD were younger (mean age 64 vs. 72 years, $p=0.017$ ) as compared to $\mathrm{AD}$ subjects.

The first main presentation symptoms were different among types of dementia (Table 2,3). Most AD patients presented with memory problems (82\%). Sixty-four (58\%) VAD patients presented with memory problems and $22 \%$ with motor symptoms. DLB patients presented with memory complaints (48\%), neuropsychiatric (17\%), and motor symptoms (17\%), while more than half of PDD patients presented with motor symptoms (57\%) followed by memory problems (18\%). FTD patients presented with behavioral change (31\%) and neuropsychiatric $(23 \%)$ and language problems $(23 \%)$.

\section{Discussion}

The prevalence of dementia increases with age. Dementia UK 2014 showed that the prevalence of dementia in the age groups of 75-79, 80-84, 85-89, and 90-94 years were $6,11,18$, and $30 \%$, respectively. The majority of dementia (>97\%) cases presented as late onset dementia [5]. Our study showed that the mean age of patients at the 
Table 2. Baseline characteristics of dementia classified by etiology

\begin{tabular}{|c|c|c|c|c|c|}
\hline Diagnosis $(n)$ & $\begin{array}{l}\text { Age at onset, } \\
\text { years }\end{array}$ & Female, $\%$ & $\begin{array}{l}\text { Education, } \\
\text { years }\end{array}$ & TMSE score & Main chief complaint (\%) \\
\hline Late onset $\mathrm{AD}(176)$ & $75(66-97)$ & 67 & $8 \pm 5$ & $18 \pm 6$ & $\begin{array}{l}\text { Memory impairment }(80) \\
\text { Neuropsychiatric problems } \\
(10)\end{array}$ \\
\hline Early onset AD (35) & $58(45-65)$ & 66 & $10 \pm 5$ & $13 \pm 6$ & Memory impairment (91) \\
\hline LV PPA (9) & $66(56-78)$ & 50 & $10 \pm 5$ & $10 \pm 7$ & $\begin{array}{l}\text { Language problems }(40) \\
\text { Memory impairment }(25)\end{array}$ \\
\hline PCA (5) & $58(56-61)$ & 60 & $8 \pm 5$ & $17 \pm 5$ & $\begin{array}{l}\text { Visuospatial problems (60) } \\
\text { Memory impairment }(40)\end{array}$ \\
\hline VAD (111) & $75(43-92)$ & 55 & $7 \pm 5$ & $16 \pm 5$ & $\begin{array}{l}\text { Memory impairment (58) } \\
\text { Motor problems (22) }\end{array}$ \\
\hline DLB (29) & $75(55-91)$ & 52 & $10 \pm 6$ & $20 \pm 4$ & $\begin{array}{l}\text { Memory impairment (48) } \\
\text { Neuropsychiatric problems } \\
\text { (17) } \\
\text { Motor problems (17) }\end{array}$ \\
\hline PDD (28) & $76(59-85)$ & 57 & $8 \pm 6$ & $20 \pm 5$ & $\begin{array}{l}\text { Motor problems (57) } \\
\text { Memory impairment (18) } \\
\text { Behavioral problems (11) }\end{array}$ \\
\hline PSP (10) & $69(58-79)$ & 46 & $9 \pm 5$ & $19 \pm 6$ & $\begin{array}{l}\text { Motor problems (50) } \\
\text { Executive problems (20) } \\
\text { Behavioral problems (20) }\end{array}$ \\
\hline MSA (4) & $64(48-75)$ & 25 & $8 \pm 6$ & $19 \pm 3$ & $\begin{array}{l}\text { Motor problems (50) } \\
\text { Executive problems (25) } \\
\text { Language problems (25) }\end{array}$ \\
\hline CBS (2) & $77(74-79)$ & 50 & $11 \pm 8$ & $15 \pm 2$ & Motor problems (100) \\
\hline Behavioral variant FTD (11) & $63(40-79)$ & 42 & $10 \pm 6$ & $22 \pm 5$ & $\begin{array}{l}\text { Behavioral problems }(34) \\
\text { Neuropsychiatric problems } \\
(24)\end{array}$ \\
\hline PPA (3) & $69(61-80)$ & 67 & $10 \pm 6$ & $14 \pm 8$ & Language problems (67) \\
\hline
\end{tabular}

Data are presented as the mean (range), mean $\pm \mathrm{SD}$, or as indicated. $\mathrm{AD}$, Alzheimer's disease; VAD, vascular dementia; LV, logopenic variant; PPA, primary progressive aphasia; PCA, posterior cortical atrophy; DLB, dementia with Lewy bodies; PDD, Parkinson's disease dementia; PSP, progressive supranuclear palsy; MSA, multiple system atrophy; CBS, corticobasal syndrome; FTD, frontotemporal dementia.

onset of dementia was 78 years, with $60 \%$ female, and most presenting with moderate dementia (mean TMSE of 17 and MOCA score of 14). The findings were consistent with a previous study in Thai dementia patients [6].

There are many causes of dementia. AD is the most common cause, although the reported prevalence varies [4-6]. Cunningham et al. [5] reviewed the pathophysiological processes of dementia and found that $\mathrm{AD}$ was the most common cause (50-75\%), followed by VAD (20\%), DLB (5\%), and FTLD (5\%). A systemic review and meta- analysis showed that the incidence of DLB was 3.8\% of new dementia diagnoses, with a prevalence of $4.2 \%$ of dementia cases in community settings and $7.5 \%$ in secondary care settings [16]. Our registry showed that AD was the most common cause of dementia (50\%), VAD was the second most common cause (24\%), followed by DLB (6\%), PDD (6\%), FTD (3\%), and PSP (2\%), which was similar to previous studies in Western patients. Some studies combined tau-associated neurodegenerative diseases, CBS, and PSP with FTD because they shared clini- 
Table 3. Baseline characteristics and symptoms at presentation in common dementia

\begin{tabular}{|c|c|c|c|c|c|c|c|c|c|}
\hline Age at diagnosis, years & $72 \pm 8$ & $75 \pm 8$ & 0.012 & $75 \pm 8$ & 0.102 & $76 \pm 6$ & 0.027 & $64 \pm 10$ & 0.017 \\
\hline \multicolumn{10}{|l|}{ Sex } \\
\hline Male & $69(33)$ & $51(46)$ & & $14(48)$ & & $12(43)$ & & $7(54)$ & \\
\hline Female & $142(67)$ & $60(54)$ & 0.023 & $15(52)$ & 0.106 & $16(57)$ & 0.302 & $6(46)$ & 0.125 \\
\hline MOCA score & $13.5 \pm 5.3$ & $12.8 \pm 5.7$ & 0.424 & $15.1 \pm 4.3$ & 0.185 & $17.2 \pm 6.2$ & 0.043 & $14.8 \pm 6$ & 0.508 \\
\hline Hypertension & $147(70)$ & $90(81)$ & 0.050 & $21(72)$ & 0.661 & $19(68)$ & 0.731 & $9(69)$ & 0.891 \\
\hline Diabetes mellitus & $65(31)$ & $32(29)$ & 0.674 & $4(14)$ & 0.066 & $6(21)$ & 0.294 & $4(31)$ & 0.980 \\
\hline Hyperlipidemia & $163(77)$ & $106(96)$ & $<0.001$ & $26(93)$ & 0.066 & $16(57)$ & 0.016 & $10(77)$ & 0.928 \\
\hline Coronary artery disease & $13(6)$ & $7(6)$ & 0.984 & $2(7)$ & 0.856 & $4(14)$ & 0.123 & - & 0.353 \\
\hline Smoking & $2(1)$ & $1(0.9)$ & 0.957 & - & 0.602 & $1(4)$ & 0.247 & - & 0.722 \\
\hline Visuospatial impairment & $3(1)$ & $3(3)$ & & - & & $1(4)$ & & - & \\
\hline Executive impairment & $3(1)$ & $4(4)$ & & $3(10)$ & & - & & $1(8)$ & \\
\hline Behavioral symptoms & $11(5)$ & $6(5)$ & & $2(6)$ & & $3(11)$ & & $4(31)$ & \\
\hline Neuropsychiatric symptoms & $17(8)$ & $10(9)$ & & $5(17)$ & & $3(11)$ & & $3(23)$ & \\
\hline Motor symptoms & $3(1)$ & $24(22)$ & $<0.001$ & $5(17)$ & $<0.001$ & $16(57)$ & $<0.001$ & $1(8)$ & $<0.001$ \\
\hline
\end{tabular}

Data are presented as the mean $\pm \mathrm{SD}$ or $n(\%)$. AD, Alzheimer's disease; VAD, vascular dementia; DLB, dementia with Lewy bodies; PDD, Parkinson's disease dementia; FTD, frontotemporal dementia.

cal frontal lobe dysfunction and pathologic tauopathy. If combining FTD, PSP, and CBS, the prevalence of tauopathy-related dementia in our registry would reach $5 \%$. The main presenting symptoms in patients with different causes of dementia were rather similar to previous reports.

Most $\mathrm{AD}$ patients presented with late onset amnestic $\operatorname{AD}(176 / 225,78 \%)$. However, $16 \%$ of the patients (35/225) presented with early onset amnestic $\mathrm{AD}, 9$ patients with LV PPA, and 5 patients with PCA. A previous review showed that early onset $\mathrm{AD}$ comprised approximately $5-6 \%$ of $\mathrm{AD}$, which had a larger genetic predisposition, more aggressive course, and presented with less memory impairment and greater involvement of other cognitive domains [17]. However, our registry showed a higher proportion of early onset $\mathrm{AD}$. This may be due to most of the registry data coming from university hospitals, where patients with atypical dementia were referred. Early onset $\mathrm{AD}$ in our registry was classified into amnestic (35 patients) and AD variants: LV PPA (4 patients), and PCA (5 patients). Early onset amnestic AD (mean age 58 years) presented with memory impairment (91\%) and the chief complaint of patients with PCA (mean age 58 years) was visuospatial problems (60\%). PCA is a neurodegenerative syndrome that primarily affects the parietal and occipital lobes, and $\mathrm{AD}$ was the most common underlying pathology [18]. The majority of PCA patients have an early age at disease onset, typically presenting between the age of 50 and 65 years. The proportion of AD patients presenting with PCA was about $5 \%$ in a specialist cognitive clinic, and up to $13 \%$ in cases of early onset AD [19, 20]. All patients with PCA in our registry, for which the diagnosis was confirmed by functional and molecular imaging, had presented with a young onset of dementia, and accounted for $11 \%$ (5/44 patients) of early onset AD cases.

YOD made up $17 \%$ of our samples. Unlike late onset dementia, YOD is slightly more prevalent in men than women. The most common cause of YOD in our registry was AD, followed by VAD and FTD. Devineni and Onyike [21] showed that the prevalence of YOD ranges from 42.8 to 68.2 per 100,000 , and the frequency of YOD in specialist clinics ranges from 7.3 to $44 \%$, depending on regions, centers in the clinical focus, local practices, referral base, and sampling methods. $\mathrm{AD}$ was also the most common cause of YOD, while the second most common cause was FTD in some studies and VAD in others.
68

Dement Geriatr Cogn Disord Extra 2021;11:64-70 DOI: $10.1159 / 000515676$
Dharmasaroja et al. 
Functional and/or molecular imaging were performed in 57 patients $(13 \%)$ in our study. The indication in most patients was atypical dementia. Functional and molecular imaging have been increasingly applied to diagnose dementia with more confidence and for early detection in mild or presymptomatic patients, especially in AD patients, where early treatment has been studied with the aim to intervene in the course of disease. Although the diagnostic criteria in other causes of dementia mainly uses clinical criteria, functional and/or molecular imaging were added as the supportive and/or indicative biomarkers. Previous studies showed that $10-30 \%$ of individuals clinically diagnosed with $\mathrm{AD}$ dementia by experts did not have an AD pathology at autopsy, and a similar portion had normal amyloid PET or amyloid beta 42 in their cerebrospinal fluid $[22,23]$. Thus, adding the results of molecular imaging, AD pathology could be diagnosed before the autopsy. However, there were some limitations. Despite the high cost of the investigation, patients are not reimbursed for these procedures in routine clinical practice in many countries, including Thailand. Moreover, adding the functional and/or molecular imaging might not help the diagnosis in some cases of dementia, such as familial prion disease or suspected non-AD pathology.

This was a large dementia registry in Thailand, using recent criteria in diagnosis and integrating the results of functional and/or molecular imaging with the clinical criteria. However, the data mainly came from the 4 participating university hospitals and 2 tertiary care hospitals, and the prevalence of each type of dementia might be different in ordinary community hospitals. This was a cross- sectional study, and some data were lacking or incomplete. Some patients did not have both TMSE and MOCA at diagnosis, but most had another test later on.

In conclusion, $\mathrm{AD}$ was the most common cause of dementia in Thai patients and the etiology of dementia and main presenting symptoms were similar to previous reports in Western patients. However, the proportion of dementia in the young was higher.

\section{Statement of Ethics}

The study was approved by the Human Research Ethics Committee of Thammasat University No. 1 (Faculty of Medicine), project No. MTU-EC-IM-0-213/60. Consent was informed and signed by the rightful relatives.

\section{Conflict of Interest Statement}

The authors have no conflicts of interest to disclose. land.

\section{Funding Sources}

This study was funded by The Neurological Society of Thai-

\section{Author Contributions}

P.A.D. and J.A.: conceptualization, methodology. P.A.D.: formal analysis, funding acquisition, writing. P.A.D., J.A., S.Po., K.J., P.Lo., M.P., L.C., C.C., and S.Pi.: investigation. All authors: review and editing.

\section{References}

1 GBD 2016 Dementia Collaborators. Global, regional, and national burden of Alzheimer's disease and other dementias, 1990-2016: a systemic analysis for the Global Burden of Diseases Study 2016. Lancet Neurol. 2019 Jan; 18(1):88-106.

2 Senanarong V, Harnphadungkit K, Poungvarin N, Vannasaeng S, Chongwisal S, Chakorn $\mathrm{T}$, et al. The Dementia and Disability Project in Thai Elderly: rational, design, methodology and early results. BMC Neurol. 2013 Jan; 13(1):3.

3 Wangtongkum S, Sucharitkul P, Silprasert N, Inthrachak R. Prevalence of dementia among population age over 45 years in Chiang Mai, Thailand. J Med Assoc Thai. 2008 Nov;91(11): 1685-90.

4 Prince M, Knapp M, Guerchet M, McCrone P, Prina M. Comas-amHerrera A, et al. De- mentia UK: update. London: Alzheimer's Society; 2014.

5 Cunningham EL, McGuinness B, Herron B, Passmore AP. Dementia. Ulster Med J. 2015 May;84(2):79-87.

6 Dharmasaroja PA, Lolekha P, Kulkantrakorn K, Charernboon T, Watcharakorn A, Piyabhan P. Natural course and predictors of severe disability and death in Thai patients with dementia. J Clin Neurosci. 2017 Dec;46:3740.

7 McKhann GM, Knopman DS, Chertkow H, Hyman BT, Jack CR Jr, Kawas CH, et al. The diagnosis of dementia due to Alzheimer's disease: recommendations from the National Institute on Aging-Alzheimer's Association workgroups on diagnostic guidelines for Alzheimer's disease. Alzheimers Dement. 2011 May;7(3):263-9.
8 Gorelick PB, Scuteri A, Black SE, Decarli C, Greenberg SM, Iadecola C, et al.; American Heart Association Stroke Council, Council on Epidemiology and Prevention, Council on Cardiovascular Nursing, Council on Cardiovascular Radiology and Intervention, and Council on Cardiovascular Surgery and Anesthesia. Vascular contributions to cognitive impairment and dementia: a statement for healthcare professionals from the american heart association/american stroke association. Stroke. 2011 Sep;42(9):2672-713.

9 Sachdev P, Kalaria R, O’Brien J, Skoog I, Alladi S, Black SE, et al.; Internationlal Society for Vascular Behavioral and Cognitive Disorders. Diagnostic criteria for vascular cognitive disorders: a VASCOG statement. Alzheimer Dis Assoc Disord. 2014 Jul-Sep; 28(3):206-18. 
10 Gorno-Tempini ML, Hillis AE, Weintraub S, Kertesz A, Mendez M, Cappa SF, et al. Classification of primary progressive aphasia and its variants. Neurology. 2011 Mar;76(11): 1006-14.

11 Rascovsky K, Hodges JR, Knopman D, Mendez MF, Kramer JH, Neuhaus J, et al. Sensitivity of revised diagnostic criteria for the behavioural variant of frontotemporal dementia. Brain. 2011 Sep;134(Pt 9):2456-77.

12 Litvan I, Agid Y, Calne D, Campbell G, Dubois B, Duvoisin RC, et al. Clinical research criteria for the diagnosis of progressive supranuclear palsy (Steele-Richardson-Olszewski syndrome): report of the NINDS-SPSP international workshop. Neurology. 1996 Jul; 47(1):1-9.

13 Armstrong MJ, Litvan I, Lang AE, Bak TH, Bhatia KP, Borroni B, et al. Criteria for the diagnosis of corticobasal degeneration. Neurology. 2013 Jan;80(5):496-503.
14 Emre M, Aarsland D, Brown R, Burn DJ, Duyckaerts C, Mizuno Y, et al. Clinical diagnostic criteria for dementia associated with Parkinson's disease. Mov Disord. 2007 Sep; 22(12):1689-707.

15 McKeith IG, Boeve BF, Dickson DW, Halliday G, Taylor JP, Weintraub D, et al. Diagnosis and management of dementia with Lewy bodies: fourth consensus report of the DLB Consortium. Neurology. 2017 Jul;89(1):88100.

16 Vann Jones SA, O’Brien JT. The prevalence and incidence of dementia with Lewy bodies: a systematic review of population and clinical studies. Psychol Med. 2014 Mar;44(4):67383.

17 Mendez MF. Early-onset Alzheimer disease and its variants. Continuum. 2019 Feb;25(1): 34-51.

18 Schott JM, Crutch SJ. Posterior cortical atrophy. Continuum. 2019 Feb;25(1):52-75.
19 Snowden JS, Stopford CL, Julien CL, Thompson JC, Davidson Y, Gibbons L, et al. Cognitive phenotypes in Alzheimer's disease and genetic risk. Cortex. 2007 Oct;43(7):835-45.

20 Koedam EL, Lauffer V, van der Vlies AE, van der Flier WM, Scheltens P, Pijnenburg YA. Early-versus late-onset Alzheimer's disease: more than age alone. J Alzheimers Dis. 2010; 19(4):1401-8.

21 Devineni B, Onyike CU. Young-onset dementia epidemiology applied to neuropsychiatry practice. Psychiatr Clin North Am. 2015 Jun;38(2):233-48.

22 Nelson PT, Head E, Schmitt FA, Davis PR, Neltner JH, Jicha GA, et al. Alzheimer's disease is not "brain aging": neuropathological, genetic, and epidemiological human studies. Acta Neuropathol. 2011 May;121(5):571-87.

23 Jack CR Jr, Bennett DA, Blennow K, Carrillo MC, Dunn B, Haeberlein SB, et al.; Contributors. NIA-AA Research Framework: toward a biological definition of Alzheimer's disease. Alzheimers Dement. 2018 Apr;14(4):535-62. 